\section{Elektronisches Rechnungsformat "ZUGFeRD“ erleichtert den Rechnungsaustausch}

Auf der CeBIT 2013 haben das Bundesministerium für Wirtschaft und Technologie (BMWi), das Bundesministerium des Innern (BMI) sowie BITKOM die Pilotanwendung der elektronischen Rechnung im Datenformat des Forums elektronische Rechnung Deutschland „ZUGFeRD“ vorgestellt. ZUGFeRD ermöglicht künftig den Austausch strukturierter Daten zwischen Rechnungssteller und Rechnungsempfänger. Das neue Datenformat ist eine wichtige Voraussetzung dafür, dass bei der Nutzung der elektronischen Rechnung bürokratische Hürden für Unternehmen und Verbraucher weiter abgebaut und Kosten gespart werden können.

Bei ZUGFeRD handelt es sich um ein übergreifendes Format für elektronische Rechnungen, das vom Forum elektronische Rechnung Deutschland (FeRD) erarbeitet wurde, an dem Verbände, Ministerien und Unternehmen beteiligt sind. Das Forum wurde unter dem Dach der vom BMWi geförderten Arbeitsgemeinschaft für wirtschaftliche Verwaltung (AWV) gegründet.

Weitere Informationen zum neuen FeRD-Datenformat sowie zur gemeinsamen Erklärung von BMWi, BMI und BITKOM zur elektronischen Rechnung sind abrufbar unter: www.ferd-net.de

\section{Verbesserte Sicherheit für Android-Systeme}

McAfee gab am 12.03.2013 bekannt, dass die erste und bisher einzige Whitelisting-Sicherheitslösung für auf Android basierende Embedded-Systeme ausgeliefert wurde. McAfee Application Control for Android ist die erste Lösung ihrer Art, die sich im Android-Kernel befindet. Über die direkte Einbettung in das Betriebssystem bietet McAfee Schutz ab der Installation oder Ausführung einer bösartigen App auf einem Android-Gerät. McAfee bietet so auch Schutz auf der Anwendungsebene von Android-Geräten.

Android wird schnell zur bevorzugten Plattform für EmbeddedIngenieure. Gemäß der "2012 Embedded Market Study“ von UBMElectronics haben 13 Prozent der Befragten Embedded-Ingenieure in 2012 ein Android-Projekt bearbeitet und 34 Prozent erwägen, in diesem Jahr mit Android zu arbeiten. Mit seinem großen Erfolg in den Wachstumsmärkten wird das Betriebssystem zunehmend zum Ziel neuer Angriffe.

Die McAfee-Embedded-Control-Lösung bietet fälschungssicheren Schutz, hohe Nutzerkontrolle und Steuerung sowie einfache Verwaltung von Android-Geräten. Bisher gab es für Embedded-Ingenieure mit Security Enhanced Linux (SELinux) nur ein Betriebssystem für durchsetzbare Sicherheitslösungen.

Vor McAfee Embedded Control funktionierten Android-Sicherheitsanwendungen nur auf Benutzerebene und die Geräte waren anfällig für Angriffe auf Systemebene. McAfee eliminiert diese Sicherheitslücke mit Hilfe einer Lösung im Kernel, um so den gesamten Android-Stack zu sichern.

McAfee Embedded Control blockiert unauthorisierte Anwendungen und Änderungen fester Funktionen sowie Point-of-Service-Infrastrukturen, einschließlich Einzelhandelsgeräte, medizinische Geräte, industrielle Steuerungssysteme, Büroausstattung, Spielgeräte, Automobil-, und verschiedene militärische Luft- und Raumfahrtgeräte.

Weitere Informationen zu McAfee Embedded Control erhalten Sie hier: http://www.McAfee.com/US/Products/Embedded-Control.aspx

\section{GI: EU-Datenschutzverordnung sollte innerbetriebliche Datenschutzkontrolle nicht aufweichen}

Der Präsidiumsarbeitskreis Datenschutz und IT-Sicherheit der Gesellschaft für Informatik e.V. (GI) fordert am 28.02.2013 die Bundesregierung und die Europäische Kommission auf, das Modells der innerbetrieblichen Kontrolle des Datenschutzes sicherzustellen, bzw. eine nationale Öffnungsklausel dafür vorzusehen. Hartmut Pohl, Sprecher des Arbeitskreises: „Der betriebliche Datenschutz in Deutschland ist ein höchst erfolgreiches Modell und sollte nicht ausgehebelt werden."

Die derzeitige Rechtslage in Deutschland sieht vor, dass Unternehmen, in denen in der Regel mindestens 10 Personen mit der automatisierten oder mindestens 20 Personen mit der nicht-automatisierten Verarbeitung personenbezogener Daten beschäftigt sind, einen betrieblichen Datenschutzbeauftragten bestellen müssen.

Der Entwurf der Europäischen Datenschutz-Grundverordnung, die nach der Vorstellung der EU-Kommission die nationalen Datenschutzgesetze in den Mitgliedsstaaten ersetzen soll, sieht dagegen vor, dass nur Unternehmen mit mehr als 250 Beschäftigten und solche, deren Kerntätigkeit in der regelmäßigen und systematischen Beobachtung von Betroffenen besteht, einen betrieblichen Datenschutzbeauftragten bestellen müssen.

- Da nur noch 0,3\% der deutschen Unternehmen betriebliche Datenschutzbeauftragte bestellen müssten, entfällt die betriebliche Kontrolle des Datenschutzes weitgehend, und es wächst die Gefahr von Datenschutzverstößen in den Unternehmen mit erheblichen Risiken für die betroffenen Bürger.

- Firmen mit mehr als 250 Mitarbeitern müssten auch dann betrieblichen Datenschutzbeauftragte bestellen, wenn sie kaum personenbezogene Daten verarbeiten ( $z$. B. Produktionsbetriebe in der Zuliefererbranche). Kleinere Firmen allerdings, deren Geschäftstätigkeit in der gewerbsmäßigen Verarbeitung personenbezogener Daten besteht, müssten dagegen keinen betrieblichen Datenschutzbeauftragten bestellen. Dies bedeutet, dass zum Teil unnötigerweise ein innerbetrieblicher Datenschutz eingerichtet wird, in anderen Fällen jedoch der unabdingbare Datenschutz fehlt.

- Die Aufsichtsbehörden müssten in den Unternehmen verstärkt kontrollieren, weil die interne Kontrolle entfällt. Dazu müssten sie personell erheblich verstärkt werden.

- Weil eine interne Kontroll- und Beratungsinstanz in Datenschutzfragen fehlt, müssten Unternehmen einen höheren Qualifikationsbedarf zum Datenschutz bei den verantwortlichen Mitarbeitern befriedigen. Daraus würden sich an die Unternehmen folgende Anforderungen zur Bestellung an die Voraussetzungen ergeben, unter denen Unternehmen betrieblichen Datenschutzbeauftragte zu bestellen haben:

- Ein Schwellenwert, der sich nur an der Anzahl der Beschäftigten eines Unternehmens orientiert, ist nicht datenschutzspezifisch und daher ohne Relevanz.

- Der Schwellenwert sollte deutlich niedriger sein und sich an der Relevanz der Verarbeitung personenbezogener Daten für die Aufgabenerfüllung der Unternehmen orientieren.

- Unternehmen, deren Kerntätigkeit in der risikobehafteten Verarbeitung personenbezogener Daten besteht, sollten ausnahmslos einen betrieblichen Datenschutzbeauftragten bestellen müssen. 